\title{
DER KURZFILM ALS (KLEINES) FORMAT
}

An einem frühen Sonntagmorgen im August 1976 raste Claude Lelouch mit einem Mercedes Benz 45oSEL 6.9 durch die Straßen von Paris. Der Film, der dabei entstand - C'était un rendez-vous ${ }^{\mathbf{1}}$ - entspricht einer ziemlich genau acht Minuten langen Plansequenz und zeigt Lelouchs waghalsige Autofahrt, die in einem Tunnel der Pariser périphérique ungefähr auf der Höhe der Metrostation Porte Dauphine beginnt und auf dem Montmartre mit Blick auf die Stadt und die Basilika Sacré-Cœur im Hintergrund endet. Um den Film im titelgebenden rendez-vous mit seiner Freundin münden zu lassen, musste Lelouch die Strecke von gut Io,5 Kilometern komme was wolle in unter zehn Minuten abgefahren haben, denn die zeitliche Länge des Films war limitiert durch die Länge von ca. 300 Metern 35mm-Farbfilm, die Lelouch gemäß eigenen Angaben vom Dreh von Si c'était à refaire ${ }^{2}$ noch übrig geblieben waren und die nun verwertet werden wollten. ${ }^{3}$

Die über das Beispiel nachvollziehbare notwendige Limitierung auf eine bestimmte Filmlänge - oder, anders gesagt, auf eine gewisse Kürze - scheint heute angesichts der Möglichkeit digitaler Filmaufnahmen auf der Produktionsebene und dem im westlichen Kontext fast flächendeckend verfügbaren Breitbandinternet (bzw. der damit verbundenen vermeintlich unbegrenzten Streamingkapazität auf Rezeptionsseite) eigentlich obsolet. Doch obwohl weder teures Filmmaterial noch Rechenleistung gespart werden müssen, erfreut sich, auch unter digitalen Vorzeichen, der Kurzfilm sowohl bei Filmemacher innen wie auch beim Publikum, vor allem im Rahmen von Filmfestivals und online, enormer Beliebtheit. ${ }^{4}$ Die medien- und filmwissenschaftliche Auseinandersetzung mit kurzen Filmen spiegelt seine immense Popularität jedoch kaum wider, was, folgt man Angela Haardt, von I990 bis 1997 Leiterin der Internationalen Kurzfilmtage Oberhausen, daran liegen mag, dass das Vokabular fehlt, um überhaupt präzise und reflektiert über den Kurzfilm zu sprechen. Haardt konstatiert zum 5o. Jubiläum des Festivals:
1 Regie: Claude Lelouch, F 1976. 2 Regie: Claude Lelouch, F 1976. 3 «Neuf minutes trente secondes de pellicule, c'est ce qui me restait à la fin du tournage de ‘Si c'était à refaire, au moment des rendus. Trouvant dommage de laisser perdre ces précieux trois cents mètres de pellicule, j'en ai profité pour réaliser un projet qui me tenait à cœur depuis longtemps [...].» Claude Lelouch, zitiert auf der offiziellen Website des Regisseurs, Les Films 13 , www.lesfilms13.com/cetait-un-rendezvous/ (1.1.2020).

4 Dies lässt sich anhand der publizierten Statistiken in Bezug auf die Filmeinreichungen und die Besucher_innenzahlen von Kurzfilmfestivals feststellen. 
5 Angela Haardt: A Different History. 50 Years of Short Film in Oberhausen, in: Katalog 50. Internationale Kurzfilmtage Oberhausen, Oberhausen 2004, 73-84, hier 73.

6 David Joselit: After Art, Princeton 2013, 52 .

7 Selbst bei den Längenangaben gehen die Definitionen und Meinungen auseinander. Ich beziehe mich hier auf die Wettbewerbsregulationen der größeren Kurzfilmfestivals im europäischen und amerikanischen Raum. Das Locarno Film Festival akzeptiert seit 2019 Filme bis 59 Minuten, Oberhausen setzt die Grenze bei 35, Rotterdam bei 60 Minuten, die Berlinale und die Internationalen Kurzfilmtage Winterthur liegen bei zo, das Sundance Festival bei 49 Minuten.

8 Eileen Bowser: The Transformation of Cinema, 1907-1915, Berkeley 1990, 191.
Kurzfilm ist ein relationaler und aus der Negation entstandener Begriff: nicht abendfüllend. Man ordnet ihn verschiedenen Genres zu, scheitert aber an den Unzulänglichkeiten der mageren Begriffsbildung für das Medium Film allgemein und der Vielfältigkeit der kurzen Formen im Besonderen. So hat er nie eine kongruente Geschichte ausbilden können, zerfällt in Einzelgeschichten von Genres und deren Untergruppierungen, wird Nationalgeschichten zugeschlagen (und da meist vernachlässigt); oder es bilden sich - in der Zwischenzeit ganz den globalen Trends folgend - unerwartete Zusammenhänge in Filmszenen aus, die international vernetzt sind, aber möglicherweise die Nachbarproduktion, die einem anderen Kontext angehört, nicht mehr wahrnehmen. ${ }^{\mathbf{5}}$

Diese «Unzulänglichkeiten der mageren Begriffsbildung» - zusätzlich zur Tatsache, dass kurze Filme selten einem Publikum (Film- und Medienwissenschaftler_innen inbegriffen) außerhalb der zeitlich beschränkten Festivalauswertung bekannt werden und nur ein Bruchteil der Gesamtproduktion in öffentlichen Videotheken oder Archiven abrufbar ist - haben Konsequenzen für die Bedeutung des Kurzfilms im akademischen Kontext, wo er aufgrund seiner schweren Fassbarkeit vernachlässigt wird. Versteht man den Kurzfilm als Format nach David Joselit, d.h. als «constellation of links» und als «a heterogeneous and often provisional structure that channels content», ${ }^{6}$ verschiebt sich das Erkenntnisinteresse vom einzelnen Film als Analyseobjekt zu Fragen der Zirkulation, der Rezeption und der diskursgeschichtlichen Positionierung von kurzen Filmen zwischen Kunst und Kino, da der Kurzfilm - dessen <Länge> die normative Dauer einer Filmvorführung unterschreitet - in einem programmatischen Kontext kaum je für sich alleine steht.

Vom frühen Stummfilm über den sogenannten Visitenkarten-Kurzfilm, der potenziellen Produzent_innen oder Investor_innen das Können einer_eines jungen Filmschaffenden unter Beweis stellen soll, bis zum kurzen Werk, das zwischen Filmfestival und Galerie zirkuliert: Unter einem Kurzfilm versteht man üblicherweise jeden Film, der auf einer Leinwand oder einem Monitor gezeigt werden kann, solange er nicht länger als I 5, 30, 6o Minuten dauert. ${ }^{7}$ Der Kurzfilm mag seine historischen Wurzeln im ersten Film, der je vorgeführt wurde, haben - trotzdem wurde er nicht I895 geboren. Der Kurzfilm existiert als Kategorie erst seit dem Moment, in dem feature film und one-reel, d.h. verschiedene Filmformate, voneinander unterschieden wurden. Damit einher ging die sofortige Unterordnung seines Stellenwerts gegenüber dem längeren Film, was auch die Bezeichnung feature, also die spezielle Qualität oder das herausgehobene Attribut von etwas, anzeigt. «A feature film was a film that cost more to make, more to buy, more to rent, and sometimes, even though not always, it cost more to see. That usually meant longer films and after 1909 <feature> was the term generally used for any multireel film», schreibt Eileen Bowser. ${ }^{8}$ Der Kurzfilm etabliert sich parallel zur kommerziellen Filmindustrie, begünstigt u. a. durch die Entwicklung von kostengünstigen Schmalfilm-Formaten. Sowohl die historische Avantgarde in den I92oer und 3oer Jahren wie auch die Nachkriegsavantgarde experimentierte mit kurzen Filmen und läutete so jeweils frühe Blütezeiten des Kurzfilms 
abseits vom Familien-, Amateur- und Gebrauchsfilm ein. In den r96oer Jahren verlor der Kurzfilm, der bis zu diesem Zeitpunkt als Vorfilm im Kino eine breite Öffentlichkeit erreicht hatte, endgültig seine Daseinsberechtigung als Teil der zu diesem Zeitpunkt dominanten Auswertungsform Kino. Ist im Folgenden von Kurzfilmen oder kurzen Filmen die Rede, beziehe ich mich daher explizit auf Werke, die nicht der normativen Länge für eine Kinoauswertung entsprechen und historisch in Nummernprogrammen - aktuell in Kurzfilmprogrammen hauptsächlich bei Festivals, im Fernsehen oder als Spezialvorführungen im Kino - als vorrangigem öffentlichem Präsentationsmodus gezeigt werden. Als Format gedacht ist der Kurzfilm kein audiovisuelles Artefakt, das für sich alleine steht, sondern ein Element innerhalb einer spezifischen Verhältnisbestimmung, bei der stets die internen Verweise, die das Werk mit sich trägt, sowie die weiteren Elemente innerhalb des Programms und auf übergeordneter Ebene auch die verhältnisbestimmende Institution mitbedacht werden müssen.

Der zu Beginn erwähnte Kurzfilm C'était un rendez-vous verhandelt den Formatbegriff im funktionalen Sinne, d.h. als Verhältnis verschiedener Parameter der Bemessung - in diesem spezifischen Fall zwischen der Länge des Filmstreifens, der Distanz einer Route und der Geschwindigkeit eines Autos. Für die Bestimmung des <Formats Kurzfilm〉als dynamischem Begriff zur Beschreibung heterogener Inhalte in kurzer Form sind die werkästhetischen, technischen oder narrativen Gegebenheiten eines Kürze-Imperativs weniger von Interesse als dessen Implikationen in Bezug auf seine Distribution und Präsentation. Ich beziehe mich hier wie bereits erwähnt explizit auf den Kunsthistoriker David Joselit, dessen Formatbegriff, wie er ihn in After Art (2013) entwickelt, maßgebend ist für eine Theorie des Kurzfilms als Format. Joselit interessiert sich weniger für die eigentliche Produktion von Bildern, ${ }^{9}$ sondern vielmehr dafür, was $<$ danach> kommt, nämlich für die Bedingungen und Auswirkungen ihrer Zirkulation in heterogenen Netzwerken. Sein Formatbegriff wird von Metaphern aus dem Feld der digitalen Zirkulation gestützt: links, connection, network etc. Das Internet, genauer das Web 2.0 und damit verbunden die explosiv wachsende Anzahl an Bildern und ihre Verbreitung, dient Joselit als Denkfigur. Er geht bei seinem Formatbegriff nicht von einem (Kunst-)Objekt aus, sondern von einem Netzwerk, das auf verschiedenen Ebenen - von der Produktion bis hin zur Rezeption - Verhältnisse herstellt zwischen dem Kunstwerk und seiner Umwelt unter den Bedingungen seiner Zirkulation:

The artwork almost always contains vestiges of what might be called the roots - or infrastructural extensions - of its entanglements in the world. These might include the means of production of the image, the human effort that brought it into being, its mode of circulation, the historical events that condition it, etc. The artwork's format solidifies and makes visible that connective tissue, reinforcing the idea that the work of art encompasses both an image and its extensions. The term format [...] points to how an image is situated within a set of relations that condition how efficacious it may be. Formats attract attention and exercise power. ${ }^{\mathbf{1 0}}$
9 Unter einem Bild versteht Joselit eine Einheit visueller Informationen, die eine Vielfalt von Formaten - im technischen Sinne - annehmen kann. 10 David Joselit: Against Representation. In conversation with David Andrew Tasman, in: DIS Magazine, 2015, dismagazine.com/ blog/75654/david-joselit-againstrepresentation/ (3.11.2019). 
11 Joselits Ausweitung des Währungsbegriffs über das Feld des Ökonomischen hinaus auf die Dimensionen des Kulturellen, Sozialen oder auch Symbolischen lässt sich auf den Kapital-Begriff, wie ihn Pierre Bourdieu bestimmt, zurückführen.

12 Joselits überspitzte Analyse dieser für ihn regressiven Form der Kunstanalyse: «Assigning a meaning is merely another way of setting an artwork's price in the currency of knowledge, transforming it into a certain kind of commodity for collectors to buy and for museums to 'sells to their audiences", Joselit: After Art, $45 \mathrm{f}$.
Das Format eines Kunstwerks hat keine Vergegenständlichung zur Folge, sondern besteht in der Art und Weise, wie ein Kunstwerk - ein Bild, ein Film, ein Buch - sich in der Welt verhält, in welchen Verhältnissen, auch der Macht und Abhängigkeit, es steht. Der Kernpunkt von Joselits Theorie zum Format liegt in der politischen Dimension, in der Art und Weise, wie Formate wirkungsmächtig werden. Joselit spricht in diesem Zusammenhang von der Währung, die sich in einem System etabliert, in dem Dynamiken des Austausches und der Bestimmung von Werteverhältnissen dominant sind. Dabei denkt er die Währung nicht nur im monetären Sinne, sondern auch als politische, kulturelle und soziale Größe." After Art liest sich stellenweise auch als ideologisch geprägtes Manifest für einen gerechteren oder zumindest demokratischeren Zugang zu Bildern und den - nicht nur monetären - Währungen, für die sie stellvertretend stehen. Indem Formate die Geschwindigkeit der Zirkulation und die Auffindbarkeit von Bildern regulieren, bestimmen sie maßgeblich auch deren 〈Währung>. Der Kurzfilm eignet sich als <kleines> Format, das sich u. a. durch oft stark komprimierte Datenmengen oder Materialanforderungen sowie eine kurze Spieldauer auszeichnet. Die dadurch gewonnene Freiheit in Bezug auf den Umlauf, die Verbreitung, das Teilen und die Kombinationsmöglichkeiten in einem breiten Spektrum von Auswertungskanälen ermöglicht es, Fragen zur Zirkulation von Filmen auszuhandeln.

Im Folgenden möchte ich die Arbeit des US-amerikanischen Filmemachers und Künstlers James N. Kienitz Wilkins als Beispiel dafür vorstellen, wie Joselits Formatbegriff medienreflexiv aufgegriffen werden kann. Kienitz Wilkins' Kurzfilme setzen sich mit der Wirkungsmacht und der Währung von zirkulierenden Bildern auseinander. Erstmals in der internationalen Kurzfilmszene auf sich aufmerksam gemacht hat Kienitz Wilkins 2014/I 5 mit der Andre-Trilogie bestehend aus Special Features (USA 20I4), Tester (USA 20I5) und abschließend B-Roll with Andre (USA 2015). Alle drei Filme versuchen mittels gestellter Interviews oder eingekauftem Material (stock imagery oder private Aufnahmen, die auf Datenträgern online verkauft wurden), die ominöse Figur Andre, den die Zuschauer_innen nie direkt zu Gesicht bekommen, zu erfassen. Im Rahmen der Trilogie untersucht und hinterfragt Kienitz Wilkins einerseits die vorherrschenden Produktionsbedingungen der Filmindustrie - in welcher der Kurzfilm aufgrund seines marginalen ökonomischen Status häufig gar nicht mitbedacht wird - und andererseits interessieren ihn Fragen nach der filmischen Repräsentation und ihrem Bezug zum Wissen. Speziell in B-Roll with Andre problematisiert der Regisseur die Annahme, dass das, was wir sehen, hören, spüren - alles, was erfahrbar ist -, nicht immer schon durch normative Vorgaben vor-formatiert ist, um Erkenntnis vermittelbar zu machen. Er verweigert ostentativ die Verknüpfung von Bildern mit einer spezifischen Bedeutung: ${ }^{12}$ Sowohl auf der Ebene der Bilder wie auch auf der der gesprochenen Sprache bleibt den Zuschauer_innen ein abschließendes Urteil über den Charakter von Andre und darüber, ob er tatsächlich einen Mord begangen hat, verwehrt. Der Kurzfilm stellt die 
Normalisierung von Wissensgenerierung in der Auseinandersetzung mit einem Film in Frage, indem er ihre Formatiertheit ausstellt und der Idee eines reinen, eines unbeschriebenen Filmbildes gegenüberstellt - eines Bildes ohne Format. $B$ Roll with Andre thematisiert also die mit Joselit erwähnte Ausdehnung über das eigentliche Bild hinaus, das Bindegewebe, wie man im Anschluss an Joselits Begriff des entan-

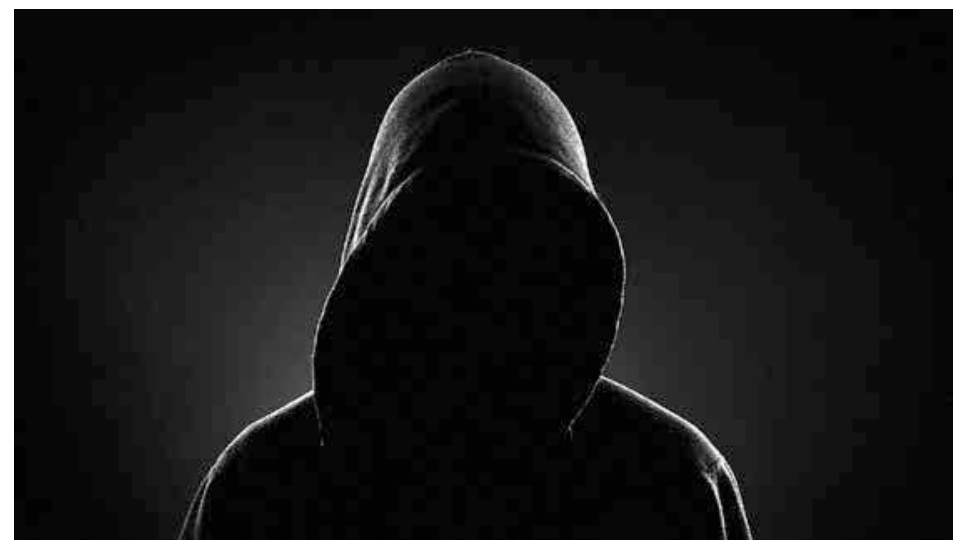
glement formulieren könnte. Dieses setzt sich zusammen aus Informationen zur Intention der Künstler_innen und den historischen Gegebenheiten, der Produktion von Bildern und ihrer Verbreitung und Rezeption. Auf der Bildebene oft höchst simpel gestaltet, bekommen

Abb. 1 B-Roll with Andre, Regie: James N. Kienitz Wilkins, USA 2015 (Orig. in Farbe)

Kienitz Wilkins' Filme durch den starken Fokus auf gesprochene Sprache einen performativen Charakter. Die Filme wurden in sukzessive höher auflösenden digitalen Formaten (zuerst BetaSP, dann MiniDV, schließlich HD) gedreht bzw. als footage auf Amazon oder eBay eingekauft. Nicht der abwesende Protagonist Andre entwickelt sich als Figur, sondern seine Repräsentation, medienreflexiv dargestellt über die Wahl des Drehformats. Den Grund für den technischen Fortschritt bei der Auswahl des Drehformats wird im letzten Kurzfilm der Trilogie evident: In B-Roll with Andre interessiert sich Kienitz Wilkins für die Idee einer reinen, ungefilterten und unvoreingenommenen Darstellung, die er mit dem höchsten Grad der Bildauflösung verknüpft. In einer Art Augenzeugenbericht, der von einem mysteriösen Mann in einem Kapuzenpulli abgelegt wird, der mit Andre Zeit im Gefängnis verbracht hat, erfahren wir von Andres Besessenheit von Bildschärfe.

B-Roll with Andre präsentiert sich selbst als Zusammenschluss von Bildmaterial aus unterschiedlichsten Quellen und von unterschiedlichster Qualität. Im Zentrum steht ein Loop aus stock footage, den Kienitz Wilkins für rund 40 Dollar im Internet erworben hat und der mit dem Titel «mysterious thug» ein mediales Gangsterklischee bedient. Von niedrigauflösenden Bildern von Google Street View und CCTV über GoPro- und iPhone-Material bis hin zur kommerziellen Inszenierung von ${ }_{4} \mathrm{~K}$-Kameras und mit einer ganzen Reihe an Versatzstücken und Zitaten aus der Film- und Kunstwelt wird der Film zu einem Streifzug durch unsere zeitgenössische Bilderwelt. Wilkins interessiert sich für den Einfluss, den die konstante Verfügbarkeit von Bildern und der Überfluss an audiovisuellem Material auf künstlerische und soziale Praktiken haben. Das Bedeutsame an diesen Bildern ist nicht unbedingt ihr Inhalt oder ihre Ästhetik, sondern eher die Reflexion darüber, woher sie stammen und wie allgegenwärtig sie verfügbar sind, wie sie in Netzwerken miteinander verbunden sind und wie sie rezipiert 
werden. Joselit hat als Ergänzung zu seinem Formatbegriff den Terminus «Epistemology of Search» eingeführt. ${ }^{13}$ So wird darauf verwiesen, dass in der heutigen digitalen Bilderwelt, deren ausschlaggebendes Merkmal die Überproduktion ist, nicht so sehr die Herstellung von neuen Bildern einen ästhetischen oder epistemologischen Wert hat, sondern deren Recherche und anschließender Zusammenschluss in neuen Zusammenhängen: $\ll[W]$ hat matters more in our contemporary digital world is not making content, but configuring it, searching for it, finding what you need and making meaning from it.» ${ }^{14}$ Der Erzähler in $B$-Roll with Andre adressiert diesen Zusammenhang zwischen Bildern und deren Bedeutung durch Auffindung und Neukontextualisierung ausdrücklich, wenn er anmerkt, dass im Film verwendete GoPro-Aufnahmen, die als Indizienbeweise in Andres Gerichtsfall behandelt werden, weder ohne Zweifel Andre als Täter zugeordnet werden können, noch in sich irgendetwas bedeuten. Erst die Platzierung in einem gewissen Kontext, der - so der Subtext seiner Erzählung - in diesem Fall bereits durch ein soziales Machtgefälle zwischen weißer Polizei und Schwarzem Täter vorbelastet ist, gibt den Bildern Bedeutung. Andre, so der Erzähler, fühlte sich stets «misrepresented $\gg .{ }^{15}$ Die Lösung für dieses Problem sind laut Andre Bilder, die nicht «formatiert», die nicht Teil einer Verhältnisbestimmung sind, sondern für sich alleine stehen:

He [Andre] was crazy about the promises of new technology. Imagine a camera that doesn't have a focus, a camera with no single point of focus, it takes it all in. And imagine it's so confident ... So resolute ... It doesn't even have a resolution. It takes the world as it comes, the entire world for the taking. ${ }^{\mathbf{1 6}}$

Mit zunehmender Bildauflösung übersteigen die Informationen das, was das menschliche Auge verarbeiten kann. Andres Vision stellt in Aussicht, dass die Überwindung der «standard definition» eine Form der Wahrnehmung erlaubt, die nicht mehr durch ein Format überhaupt greifbar gemacht wird, sondern reine, unformatierte Wahrnehmung möglich macht. Während man für einige Momente wieder das eingekaufte Bildmaterial des gesichtslosen Erzählers sieht, verbindet dieser die Frage nach dem Wert von Bildern mit der Referenz auf Tugenden wie Wahrheit und <Gut>-Sein:

13 Ebd., 56.

14 David Joselit: The Epistemology of Search. An Interview with David Joselit, in: A.R.P.A Journal, 13.11.2014, www.arpajournal.net/the-epistemologyof-search (11.9.2019).

15 James N. Kienitz Wilkins: B-Roll with Andre [persönliches Transkript des Filmemachers].

16 Ebd.
Sometimes he'd [Andre] go on and on about resolutions. Being a resolute person. Had this thing about resolutions in digital cameras, like there's a moral reason to pursue the higher resolutions. You actually become a better person. Like according to Plato, you know, Plato's divided line ... There's the visible world, with its shadows, reflections, and images ... All that crap is subpar. It's all belief and opinion. The appearances of things. Think about the camera your mom gave you in I999: It's all illusion, according to Andre. That's just standard. Standard definition. But then we move higher, getting into the HD. High definition. What feels like the physical world comes to life. But still. It's still an illusion, he would say. Why? Cause there's something better, something not limited by our vision and our senses. So OK. Now what? Now there's $2 \mathrm{~K},{ }_{4} \mathrm{~K}, 6 \mathrm{~K}$ resolution. Now we start getting mathematical. Our eyes can't take in all the information, it's not about our eyes anymore. It's about 
thinking. Calculating the benefits. But yo, something's amiss. We need to get to the highest level: Understanding. Pure ideas, pure Form. The Good that is Good unto itself. That's what Andre wanted. It's where he wanted to be. He was crazy about the promises of new technology. [...] Probably why he was so fucking frustrated, why he was so impatient, why he was so dangerous. He wanted to be Good. ${ }^{17}$

Wert, hier in einen moralischphilosophischen Kontext gestellt,

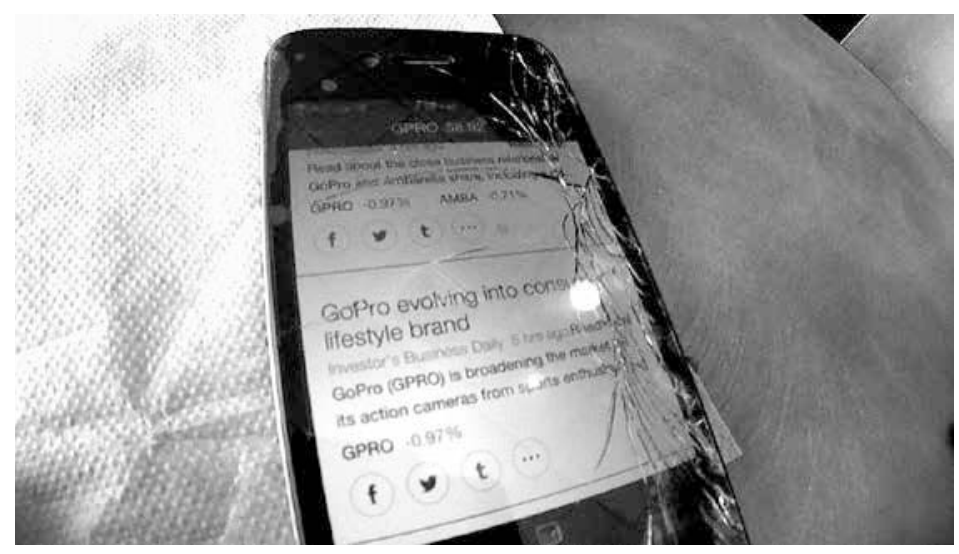
wird in B-Roll with Andre mit technischen Formaten verknüpft. Denkt man über dieses konkrete Beispiel hinaus generell an den Kurzfilm als Format, so formen die Muster von Verbindungen und Links, die Kienitz Wilkins' Film auffächert, ein Beziehungsgefüge mit externen sozialen, kulturellen, politischen und ökonomischen Faktoren. Der Regisseur selbst reflektiert die soziopolitische Ebene seines Schaffens in Bezug auf diese Dimensionen:

\begin{abstract}
Beyond aesthetics, the more important thing to me is the political dimension of access. I don't have any money. I make films. What do I do? How do I self-define? [...] So the pontifications of the characters have direct real-world relationship to the restrictions and challenges and fantasies within my own practice. I'm not saying these characters are me, but to avoid talking about what is directly in front of us - the image-making vessels, the power structures, relationship to money, delegation of labor, branding, construction of impossible fantasies, the things that are overlooked or obnoxious - is not only false, but just boring. And if I ever get rich, I expect to be very boring. ${ }^{18}$
\end{abstract}

B-Roll with Andre ist Teil einer Trilogie von Filmen, die Bezüge untereinander herstellen und aus Bildmaterial aus unterschiedlichen Quellen und mit unterschiedlichen Drehformaten bestehen. Über die Aneinanderreihung von technischen Formaten und der Narration eines Erzählers verhandelt der Kurzfilm verschiedene Beziehungsgefüge: Die Verbindungen - Links - zwischen Bildauflösung und Wahrheit. Die Verbindung zwischen Bildern und Wissen. Die Verbindung zwischen Wissen und Repräsentation. Die Verbindung zwischen Repräsentation und Zugang zu Kameras, die Repräsentation inszenieren. Die Verbindung zwischen der big-cinema-Industrie und der prekären Existenz als Kurzfilmemacher. Das ist nicht die Bedeutung des Films, sondern sein Format.
Abb. 2 B-Roll with Andre, Regie: James N. Kienitz Wilkins, USA 2015 (Orig. in Farbe)

17 Ebd.

18 James N. Kienitz Wilkins: James N. Kienitz Wilkins in Conversation with Herb Shellenberger, in: The Brooklyn Rail, März 2016, brooklynrail.org/2016/03/film/james-nkienitz-wilkins-with-herb-shellenberger (4.12.2019). 